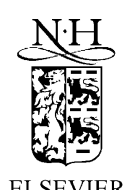

Nuclear Physics B 612 [FS] (2001) 461-478

www.elsevier.com/locate/npe

\title{
Integrable open boundary conditions for the Bariev model of three coupled $X Y$ spin chains
}

\author{
A. Foerster ${ }^{\text {a }}$, M.D. Gould ${ }^{\text {b }}$, X.-W. Guan ${ }^{\text {a }}$, I. Roditi ${ }^{\text {c,d }}$, H.-Q. Zhou ${ }^{\text {b }}$ \\ a Instituto de Fisica da UFRGS, Av. Bento Goncalves, 9500, Porto Alegre, 91501-970, Brazil \\ $\mathrm{b}$ Department of Mathematics, University of Queensland, Brisbane, Qld 4072, Australia \\ ${ }^{c}$ Centro Brasileiro de Pesquisas Fisicas, Rua Dr. Xavier Sigaud 150, 22290-180, Rio de Janeiro-RJ, Brazil \\ ${ }^{\mathrm{d}}$ C.N. Yang Institute for Theoretical Physics, State University of New York at Stony Brook, \\ Stony Brook, NY 11794-3840, USA
}

Received 8 May 2001; accepted 17 July 2001

\begin{abstract}
The integrable open-boundary conditions for the Bariev model of three coupled one-dimensional $X Y$ spin chains are studied in the framework of the boundary quantum inverse scattering method. Three kinds of diagonal boundary $K$-matrices leading to nine classes of possible choices of boundary fields are found and the corresponding integrable boundary terms are presented explicitly. The boundary Hamiltonian is solved by using the coordinate Bethe ansatz technique and the Bethe ansatz equations are derived. ๑ 2001 Elsevier Science B.V. All rights reserved.
\end{abstract}

PACS: 71.10.-w; 71.10.Fd; 75.10.Jm

Keywords: Integrable spin chains; Algebraic Bethe ansatz; Yang-Baxter algebra; Reflection equations

\section{Introduction}

Since the discovery of high temperature superconductivity in cuprates [1], a tremendous effort has been made to uncover a theoretical framework capable of explaining this amazing phenomenon. It is a general belief that the properties of strongly correlated electron systems showing a non-Fermi liquid behaviour are closely related to those materials showing high $T_{c}$ superconductivity. This has caused an increasing interest in strongly correlated electron models [2-5]. Integrable models that have been widely studied include the 1D Hubbard model solved by Lieb and Wu [6] and the supersymmetric t-J model $[7,8]$. Another model with special features relevant for high $T_{c}$ superconductivity is the 1D Bariev $[9,10]$, as it exhibits the existence of hole pairs of Cooper type.

E-mail address: guan@if.ufrgs.br (X.-W. Guan). 
On the other hand, important progress in the realm of completely integrable systems is the generalization of the usual quantum inverse scattering method (QISM) [11-13] to incorporate open boundary conditions that preserve integrability [14-17]. The presence of the integrable boundaries leading to a pure back-scattering on each end of a quantum chain results in rich physical phenomena [18-23]. It has been clarified that 1D quantum systems with boundary fields are closely related to impurity problems. Also due to their connection to the Kondo problem and boundary conformal field theory in low-dimensional quantum many-body systems, the integrable boundaries could be a useful nonperturbative way to investigate the impurity effects in the condensed matter physics. The prototypical 1D Hubbard model and t-J model have been thoroughly investigated with boundary impurities and boundary fields [18-25]. Subsequently, other strongly correlated electron systems, such as the 1D Bariev model of two coupled $X Y$ spin chains have been partially studied and the reflections equations (RE) solved [26]. In that case, a new class of boundary reflection $K$-matrices for the model lead to pure magnetic boundary fields in the Hamiltonian which may have a feasible realization by applying boundary external fields in experiments on quantum wires. An interesting aspect here is that after Jordan-Wigner transformation, the coupled $X Y$ spin chains can be presented as correlated electron systems where the hopping terms depend on the occupation numbers at sublattices. This is thought to be useful in studying conductivity properties in such non-Fermi liquids. Along this line, the integrability and boundary conditions for a Bariev model of three coupled $X Y$ chains has been studied in the frame work of the QISM by Zhou and coworkers [27,28] recently. However, we have good reasons to expect that the model would permit other kinds of integrable boundary terms associated with some new solutions to the RE. We revisit the model seeking a complete understanding of the open boundary conditions for the model. As noticed in [28] the quantum $R$-matrix of the model we study does not possess the crossing-unitarity. This causes a violation of the isomorphism between $K_{+}$and $K_{-}$matrix which satisfy two reflection equations (RE) separately. The integrability at left and right boundaries demand rather complicated RE which in turn expose bulk symmetries on the left and right boundary terms. In order to maintain the integrability of models where the $R$-matrix does not possess the crossing-unitarity property some new objects have to be introduced. It is found that the three coupled $X Y$ chains permits three kinds of $K$-matrices to each RE which lead to nine classes of integrable boundary terms containing the ones for the two coupled $X Y$ spin chain as special cases. These integrable boundary terms containing different on-site Coulomb interactions and chemical potentials reveal the symmetry of exchanging the sublattices. We derive the Hamiltonian of the model with nine classes of boundary terms from the expansion of the boundary transfer matrix around the zero spectral-parameter point up to the fourth, third and second orders, respectively. Furthermore, we solve the boundary model by means of the coordinate Bethe ansatz method and derive the Bethe ansatz equations.

The paper is organized as follows. In Section 2 we construct the Bariev model of three coupled $X Y$ spin chains with nine classes of boundary fields by means of the QISM adapted to special boundary conditions. The basic quantities, e.g., the $R$-matrix, the matrices $K_{ \pm}$defining the boundary terms, the monodromy matrices and the transfer 
matrices are defined. In particular, we solve two RE separately and obtain three independent classes of solutions to each REs using the variable-separation method. The relation between the transfer matrices and the Bariev Hamiltonians with different boundary fields is established. Section 3 is devoted to the solution of the models through coordinate Bethe ansatz method. Section 4 presents our conclusions.

\section{Boundary $K$-matrices for the coupled spin chains}

We consider a spin chain model defined by the following Hamiltonian

$$
H=\sum_{j=1}^{L-1} H_{j, j+1}+B_{1}^{(m)}+B_{L}^{(l)}
$$

where $H_{j, j+1}$ denotes the bulk Hamiltonian density of three $X Y$ spin chains coupled to each other [27]

$$
\begin{aligned}
& H_{j, j+1}=\sum_{\alpha}\left(\sigma_{j(\alpha)}^{+} \sigma_{j+1(\alpha)}^{-}+\sigma_{j(\alpha)}^{-} \sigma_{j+1(\alpha)}^{+}\right) \\
& \times \exp \left[\eta \sum_{\alpha^{\prime} \neq \alpha} \sigma_{j+\theta\left(\alpha^{\prime}-\alpha\right)\left(\alpha^{\prime}\right)}^{+} \sigma_{j+\theta\left(\alpha^{\prime}-\alpha\right)\left(\alpha^{\prime}\right)}^{-}\right],
\end{aligned}
$$

where $\sigma_{j(\alpha)}^{ \pm}=\frac{1}{2}\left(\sigma_{j(\alpha)}^{x} \pm i \sigma_{j(\alpha)}^{y}\right)$ with $\sigma_{j(\alpha)}^{x}, \sigma_{j(\alpha)}^{y}, \sigma_{j(\alpha)}^{z}$ being the usual Pauli spin operators at site $j$ corresponding to the $\alpha$ th $(\alpha=1,2,3) X Y$ spin chain, $\theta\left(\alpha^{\prime}-\alpha\right)$ is a step function of $\left(\alpha^{\prime}-\alpha\right)$ and $\eta$ is a coupling constant; $B_{1}$ and $B_{L}$ are left and right boundary terms of the form

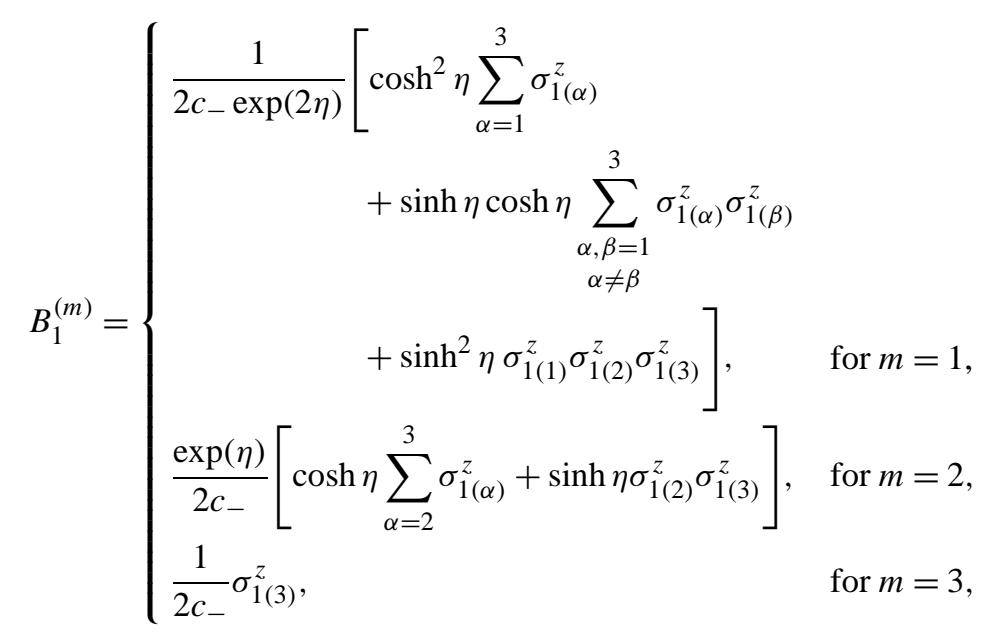




$$
B_{L}^{(l)}=\left\{\begin{array}{cc}
\frac{1}{2 c_{+}}\left[\cosh ^{2} \eta \sum_{\alpha=1}^{3} \sigma_{L(\alpha)}^{z}\right. & \\
& +\sinh \eta \cosh \eta \sum_{\substack{\alpha, \beta=1 \\
\alpha \neq \beta}}^{3} \sigma_{L(\alpha)}^{z} \sigma_{L(\beta)}^{z} \\
\left.+\sinh ^{2} \eta \sigma_{L(1)}^{z} \sigma_{L(2)}^{z} \sigma_{L(3)}^{z}\right], & \\
\frac{1}{2 c_{+} \exp (\eta)}\left[\cosh \eta \sum_{\alpha=1}^{2} \sigma_{L(\alpha)}^{z}+\sinh \eta \sigma_{L(1)}^{z} \sigma_{L(2)}^{z}\right], & \text { for } l=2, \\
\frac{1}{2 c_{+}} \sigma_{L(1)}^{z}, & \text { for } l=3,
\end{array}\right.
$$

where $c_{ \pm}$are parameters describing boundary effects. It is worth mentioning that the boundary terms containing three- and two-spin interactions and chemical potentials at the left and right ends are consistent with the bulk symmetry which is a combination of the inversion $j \rightarrow L-j+1$ and the exchange among the sublattices. With the different choices of the pair $(m, l) m, l=1,2,3$, it appears that there exist nine classes of integrable boundary terms compatible with the integrability of the model. After a generalized JordanWigner transformation, Hamiltonian (2.1) becomes a strongly correlated electronic system with boundary interactions. Quite remarkably, if one restricts the Hilbert space to the one which only consists of, say, $\sigma_{(1)}, \sigma_{(2)}$, then Hamiltonian (2.1) reduces to that of two coupled $X Y$ open chains with special boundary interactions, which has been considered in [26].

We now establish the quantum integrability for the system defined by Hamiltonian (2.1), by using the general formalism described in the paper [17]. To fix notation, let us briefly recall some basic quantities for the bulk model (2.2) with periodic boundary conditions. As was shown in [27], the bulk model Hamiltonian commutes with a one-parameter family of bulk transfer matrix $\tau(u)$ of a two-dimensional lattice statistical mechanics model. This transfer matrix is the trace of a monodromy matrix $T(u)$, which is defined, as usual, by

$$
T(u)=L_{0 N}(u) \cdots L_{01}(u)
$$

with $L_{0 j}(u)$ of the form,

$$
L_{0 j}(u)=L_{0 j}^{(1)}(u) L_{0 j}^{(2)}(u) L_{0 j}^{(3)}(u),
$$

where

$$
\begin{aligned}
& L_{0 j}^{(\alpha)}(u)= \frac{1}{2}\left(1+\sigma_{j(\alpha)}^{z} \sigma_{0(\alpha)}^{z}\right)+\frac{1}{2} u\left(1-\sigma_{j(\alpha)}^{z} \sigma_{0(\alpha)}^{z}\right) \exp \left(\eta \sum_{\substack{\alpha^{\prime}=1 \\
\alpha^{\prime} \neq \alpha}}^{3} \sigma_{0\left(\alpha^{\prime}\right)}^{+} \sigma_{0\left(\alpha^{\prime}\right)}^{-}\right) \\
&+\left(\sigma_{j(\alpha)}^{-} \sigma_{0(\alpha)}^{+}+\sigma_{j(\alpha)}^{+} \sigma_{0(\alpha)}^{-}\right) \\
& \sqrt{1+\exp \left(2 \eta \sum_{\substack{\alpha^{\prime}=1 \\
\alpha^{\prime} \neq \alpha}}^{3} \sigma_{0\left(\alpha^{\prime}\right)}^{+} \sigma_{0\left(\alpha^{\prime}\right)}^{-}\right) u^{2}}
\end{aligned}
$$


The commutativity of the bulk transfer matrices $\tau(u)$ for different values of the spectral parameter $u$ follows from the fact that the monodromy matrix $T(u)$ satisfies the YangBaxter algebra

$$
R_{12}(u, v) \stackrel{1}{T}(u) \stackrel{2}{T}(v)=\stackrel{2}{T}(v) \stackrel{1}{T}(u) R_{12}(u, v) .
$$

The explicit form of the corresponding $R$-matrix $R_{12}\left(u_{1}, u_{2}\right)$ can be found in [27]. Here we only emphasize that the local monodromy matrix as well as the quantum $R$-matrix does not possess the crossing symmetry. It satisfies the nonadditive Yang-Baxter equation

$$
R_{12}(u, v) R_{13}(u, w) R_{23}(v, w)=R_{23}(v, w) R_{13}(u, w) R_{12}(u, v) .
$$

Following the QISM adapted to the case of special boundary conditions, we define the doubled monodromy matrix as

$$
U_{-}(u)=T(u) K_{-}(u) T^{-1}(-u),
$$

such that the boundary transfer matrix is given by

$$
\tau(u)=T r_{0} K_{+}(u) U_{-}(u),
$$

where $T^{-1}$ is the inverse of the monodromy $T$ and $K_{ \pm}$are the matrices defining the boundaries. The requirement that they obey the RE [26,28]

$$
\begin{aligned}
& R_{12}(u, v) \stackrel{1}{K_{-}}(u) R_{21}(v,-u) \stackrel{2}{K_{-}}(v) \\
& \quad=\stackrel{2}{K_{-}}(v) R_{12}(u,-v) \stackrel{1}{K_{-}}(u) R_{21}(-v,-u), \\
& R_{21}^{\mathrm{t}_{1} \mathrm{t}_{2}}(v, u) \stackrel{1}{\mathrm{t}_{+}}(u) \widetilde{R}_{12}(-u, v) \stackrel{2}{\mathrm{t}_{2}}(v) \\
& \quad=\stackrel{2}{\mathrm{t}_{+}}(v) \widetilde{R}_{21}(-v, u) \stackrel{1}{\mathrm{t}_{+}^{\mathrm{t}_{1}}}(u) R_{12}^{\mathrm{t}_{1} \mathrm{t}_{2}}(-u,-v),
\end{aligned}
$$

together with the Yang-Baxter algebra and the following properties

$$
\begin{aligned}
R_{12}(u, v) R_{21}(v, u) & =1, \\
\widetilde{R}_{21}^{\mathrm{t}_{1}}(-v, u) R_{12}^{\mathrm{t}_{2}}(u,-v) & =1, \\
\widetilde{R}_{12}^{\mathrm{t}_{2}}(-u, v) R_{21}^{\mathrm{t}_{1}}(v,-u) & =1 .
\end{aligned}
$$

assure that the transfer matrix commutes for different spectral parameters, proving the integrability of the model.

Therefore, the transfer matrix (2.11) may be considered as the generating function of infinitely many integrals of motion for the system. We emphasize that there is no isomorphism between the matrices $K_{+}(u)$ and $K_{-}(u)$, due to the absence of the crossing symmetry for the $R$-matrix. Therefore, we have to solve two REs separately in order to fix the boundaries. In Appendix A, this calculation is presented in detail using the variableseparation prescription. We find three different classes of boundary $K_{ \pm}$matrices consistent 
with the integrability of the Hamiltonian (2.1). Let us first list those related with $K_{-}$-matrix

$$
K_{-}^{(m)}(u)=\frac{1}{\lambda_{-}}\left(\begin{array}{cccccccc}
A_{-}(u) & 0 & 0 & 0 & 0 & 0 & 0 & 0 \\
0 & B_{-}(u) & 0 & 0 & 0 & 0 & 0 & 0 \\
0 & 0 & C_{-}(u) & 0 & 0 & 0 & 0 & 0 \\
0 & 0 & 0 & D_{-}(u) & 0 & 0 & 0 & 0 \\
0 & 0 & 0 & 0 & E_{-}(u) & 0 & 0 & 0 \\
0 & 0 & 0 & 0 & 0 & F_{-}(u) & 0 & 0 \\
0 & 0 & 0 & 0 & 0 & 0 & G_{-}(u) & 0 \\
0 & 0 & 0 & 0 & 0 & 0 & 0 & H_{-}(u)
\end{array}\right),
$$

where for $m=1$ :

$$
\begin{aligned}
& A_{-}(u)=\left(c_{-}+u\right)\left(e^{2 \eta} c_{-}+u\right)\left(e^{4 \eta} c_{-}+u\right), \\
& B_{-}(u)=\left(c_{-}-u\right)\left(e^{2 \eta} c_{-}+u\right)\left(e^{4 \eta} c_{-}+u\right), \\
& C_{-}(u)=\left(c_{-}-u\right)\left(e^{2 \eta} c_{-}+u\right)\left(e^{4 \eta} c_{-}+u\right), \\
& D_{-}(u)=\left(c_{-}-u\right)\left(e^{2 \eta} c_{-}-u\right)\left(e^{4 \eta} c_{-}+u\right), \\
& E_{-}(u)=\left(c_{-}-u\right)\left(e^{2 \eta} c_{-}+u\right)\left(e^{4 \eta} c_{-}+u\right), \\
& F_{-}(u)=\left(c_{-}-u\right)\left(e^{2 \eta} c_{-}-u\right)\left(e^{4 \eta} c_{-}+u\right), \\
& G_{-}(u)=\left(c_{-}-u\right)\left(e^{2 \eta} c_{-}-u\right)\left(e^{4 \eta} c_{-}+u\right), \\
& H_{-}(u)=\left(c_{-}-u\right)\left(e^{2 \eta} c_{-}-u\right)\left(e^{4 \eta} c_{-}-u\right), \\
& \lambda_{-}=\frac{1}{e^{6 \eta} c_{-}^{3}},
\end{aligned}
$$

for $m=2$ :

$$
\begin{aligned}
& A_{-}(u)=E_{-}(u)=\left(c_{-}+u\right)\left(c_{-}+e^{2 \eta} u\right), \\
& B_{-}(u)=C_{-}(u)=F_{-}(u)=G_{-}(u)=\left(c_{-}+u\right)\left(c_{-}-e^{2 \eta} u\right), \\
& D_{-}(u)=H_{-}(u)=\left(c_{-}-u\right)\left(c_{-}-e^{2 \eta} u\right), \\
& \lambda_{-}=\frac{1}{c_{-}^{2}}
\end{aligned}
$$

for $m=3$ :

$$
\begin{aligned}
& A_{-}(u)=C_{-}(u)=E_{-}(u)=G_{-}(u)=\left(c_{-}+u\right), \\
& B_{-}(u)=D_{-}(u)=F_{-}(u)=H_{-}(u)=\left(c_{-}-u\right), \\
& \lambda_{-}=\frac{1}{c_{-}} .
\end{aligned}
$$

However, it is much more tedious to find the boundary $K$-matrix $K_{+}(u)$, since not only the corresponding RE is more involved but also the new object $\widetilde{R}_{12}(u, v)$ is more 
complicated. We list the final result here and some details can be found in Appendix A,

$$
K_{+}^{(l)}(u)=\left(\begin{array}{cccccccc}
A_{+}(u) & 0 & 0 & 0 & 0 & 0 & 0 & 0 \\
0 & B_{+}(u) & 0 & 0 & 0 & 0 & 0 & 0 \\
0 & 0 & C_{+}(u) & 0 & 0 & 0 & 0 & 0 \\
0 & 0 & 0 & D_{+}(u) & 0 & 0 & 0 & 0 \\
0 & 0 & 0 & 0 & E_{+}(u) & 0 & 0 & 0 \\
0 & 0 & 0 & 0 & 0 & F_{+}(u) & 0 & 0 \\
0 & 0 & 0 & 0 & 0 & 0 & G_{+}(u) & 0 \\
0 & 0 & 0 & 0 & 0 & 0 & 0 & H_{+}(u)
\end{array}\right)
$$

for $l=1$ :

$$
\begin{aligned}
& A_{+}(u)=\left(e^{6 \eta} c_{+} u-1\right)\left(e^{4 \eta} c_{+} u-1\right)\left(e^{2 \eta} c_{+} u-1\right), \\
& B_{+}(u)=e^{4 \eta}\left(e^{2 \eta} c_{+} u+1\right)\left(e^{4 \eta} c_{+} u-1\right)\left(e^{2 \eta} c_{+} u-1\right), \\
& C_{+}(u)=e^{2 \eta}\left(e^{2 \eta} c_{+} u+1\right)\left(e^{4 \eta} c_{+} u-1\right)\left(e^{2 \eta} c_{+} u-1\right), \\
& D_{+}(u)=e^{6 \eta}\left(e^{2 \eta} c_{+} u+1\right)\left(c_{+} u+1\right)\left(e^{2 \eta} c_{+} u-1\right), \\
& E_{+}(u)=\left(e^{2 \eta} c_{+} u+1\right)\left(e^{4 \eta} c_{+} u-1\right)\left(e^{2 \eta} c_{+} u-1\right), \\
& F_{+}(u)=e^{4 \eta}\left(c_{+} u+1\right)\left(e^{2 \eta} c_{+} u+1\right)\left(e^{2 \eta} c_{+} u-1\right), \\
& G_{+}(u)=e^{2 \eta}\left(c_{+} u+1\right)\left(e^{2 \eta} c_{+} u+1\right)\left(e^{2 \eta} c_{+} u-1\right), \\
& H_{+}(u)=e^{4 \eta}\left(c_{+} u+e^{2 \eta}\right)\left(c_{+} u+1\right)\left(e^{2 \eta} c_{+} u+1\right) .
\end{aligned}
$$

for $l=2$ :

$$
\begin{aligned}
& A_{+}(u)=B_{+}(u)=\left(e^{6 \eta} c_{+} u-1\right)\left(e^{4 \eta} c_{+} u-1\right), \\
& C_{+}(u)=D_{+}(u)=e^{2 \eta}\left(e^{2 \eta} c_{+} u+1\right)\left(e^{4 \eta} c_{+} u-1\right), \\
& E_{+}(u)=F_{+}(u)=\left(e^{2 \eta} c_{+} u+1\right)\left(e^{4 \eta} c_{+} u-1\right), \\
& G_{+}(u)=H_{+}(u)=e^{2 \eta}\left(c_{+} u+1\right)\left(e^{2 \eta} c_{+} u+1\right),
\end{aligned}
$$

for $l=3$ :

$$
\begin{aligned}
& A_{+}(u)=B_{+}(u)=e^{2 \eta}\left(e^{4 \eta} c_{+} u-1\right), \\
& C_{+}(u)=D_{+}(u)=\left(e^{4 \eta} c_{+} u-1\right), \\
& E_{+}(u)=F_{+}(u)=e^{2 \eta}\left(c_{+} u+1\right), \\
& G_{+}(u)=H_{+}(u)=\left(c_{+} u+1\right) .
\end{aligned}
$$

The above explicit formulae for $K_{ \pm}(u)$, derived by solving the two REs directly, clearly show that no automorphism between $K_{+}(u)$ and $K_{-}(u)$ exists and $K_{+}(u)$ can not be obtained from $K_{-}(u)$. These three classes of boundary $K_{ \pm}$-matrices provide nine possible choices of BC, according to the combination of the boundary pairs $\left(K_{-}^{(m)}(u), K_{+}^{(l)}(u)\right)$, $m, l=1,2,3$, which originate the pair-boundary terms $\left(B_{1}^{(m)}, B_{L}^{(l)}\right)$, respectively. Taking into account the fact that $\operatorname{Tr} K_{+}^{(1)}(0)=0, \operatorname{Tr} \dot{K}_{+}^{(1)}(0)=0, \operatorname{Tr} \ddot{K}_{+}^{(1)}(0)=0$ and 
$\operatorname{Tr} K_{+}^{(2)}(0)=0, \operatorname{Tr} \dot{K}_{+}^{(2)}(0)=0$, as well as $\operatorname{Tr} K_{+}^{(3)}(0)=0$, we can show that the Hamiltonian (2.1) with the boundary terms pairs $\left(K_{-}^{(m)}(u), K_{+}^{(l)}(u)\right)$ is related to the transfer matrix (2.11) in the following way

$$
\begin{array}{lll}
\tau(u)=C_{1} u^{3}+C_{2}(H+\text { const }) u^{4}+\cdots, & \text { for } l=1, \\
\tau(u)=C_{3} u^{2}+C_{4}(H+\text { const }) u^{3}+\cdots, & \text { for } l=2, \\
\tau(u)=C_{5} u+C_{6}(H+\text { const }) u^{2}+\cdots, & \text { for } l=3 .
\end{array}
$$

Above $C_{i}, i=1, \ldots, 6$, are some scalar functions of the boundary parameters. The symbols, either "bullet" or "prime" denote the derivative with respect to the spectral parameter. For $l=1$, the Hamiltonian (2.1) is related to the fourth derivative of the boundary transfer matrix $\tau(u)$ which can be derived by the simplified formula [28]

$$
\begin{aligned}
& H \equiv \frac{\tau^{\prime \prime \prime \prime}(0)}{8 \operatorname{Tr} K_{+}^{(1) \prime \prime \prime}(0)} \\
&=\sum_{j=1}^{L-1} H_{j j+1}+\frac{1}{2} P_{01} K_{-}^{(m) \prime}(0) P_{01}+\frac{3}{\operatorname{Tr} K_{+}^{(1) \prime \prime \prime}(0)} \operatorname{Tr}\left(K_{+}^{(1) \prime \prime}(0) L_{0 L}^{\prime}(0) P_{0 L}\right), \\
& H_{j, j+1}=P_{0, j+1}(0) L_{0 j}^{\prime}(0) P_{0 j}^{-1}(0) P_{0, j+1}^{-1}(0)
\end{aligned}
$$

with $m=1,2,3$.

However, to obtain new boundary terms corresponding to the $K_{+}^{(l)}$-matrices when $l=2,3$, we have to develop new formulae regarding to the relations (2.20) and (2.21). What follows are the general formulae of the Hamiltonian related to Eqs. (2.20) and (2.21) given by

$$
\begin{aligned}
H \equiv & \frac{t^{\prime \prime \prime}(0)}{f} \\
= & \sum_{j=1}^{L-1} H_{j j+1}+\frac{1}{2} P_{01} K_{-}^{(m) \prime}(0) P_{01} \\
& +\frac{1}{f}\left\{\operatorname{Tr}\left(K_{+}^{(2)}(0) L_{0 L}^{\prime \prime \prime}(0) P_{0 L}\right)+\operatorname{Tr}\left(K_{+}^{(2)}(0) P_{0 L} \bar{L}_{0 L}^{\prime \prime \prime}(0)\right)\right. \\
& +6 \operatorname{Tr}\left(K_{+}^{(2) \prime \prime}(0) L_{0 L}^{\prime}(0) P_{0 L}\right)+3 \operatorname{Tr}\left(K_{+}^{(2) \prime}(0) L_{0 L}^{\prime \prime}(0) P_{0 L}\right) \\
& +3 \operatorname{Tr}\left(K_{+}^{(2) \prime}(0) P_{0 L} \bar{L}_{0 L}^{\prime \prime}(0)\right)+3 \operatorname{Tr}\left(K_{+}^{(2)}(0) L_{0 L}^{\prime}(0) \bar{L}_{0 L}^{\prime \prime}(0)\right) \\
& \left.+3 \operatorname{Tr}\left(K_{+}^{(2)}(0) L_{0 L}^{\prime \prime}(0) \bar{L}_{0 L}^{\prime}(0)\right)+6 \operatorname{Tr}\left(K_{+}^{(2) \prime}(0) L_{0 L}^{\prime}(0) \bar{L}_{0 L}^{\prime}(0)\right)\right\}
\end{aligned}
$$

and

$$
\begin{aligned}
H & \equiv \frac{t^{(2)}(0)}{\operatorname{Tr} K_{+}^{(3) \prime}(0)} \\
& =\sum_{j=1}^{L-1} H_{j j+1}+\frac{1}{2} P_{01} K_{-}^{(m) \prime}(0) P_{01}
\end{aligned}
$$




$$
\begin{aligned}
+\frac{1}{\operatorname{Tr} K_{+}^{(3) \prime}(0)}\{ & \operatorname{Tr}\left(K_{+}^{(2)}(0) L_{0 L}^{\prime \prime}(0) P_{0 L}\right)+\operatorname{Tr}\left(K_{+}^{(2)}(0) P_{0 L} \bar{L}_{0 L}^{\prime \prime}(0)\right) \\
& \left.+2 \operatorname{Tr}\left(K_{+}^{(2)}(0) L_{0 L}^{\prime}(0) \bar{L}_{0 L}^{\prime}(0)\right)+2 \operatorname{Tr}\left(K_{+}^{(2) \prime}(0) L_{0 L}^{\prime}(0) P_{0 L}\right)\right\},
\end{aligned}
$$

respectively. Above

$$
\begin{aligned}
& \left.\bar{L}_{0 L}^{\prime}(0) \equiv \frac{d}{d u} L_{0 L}^{-1}(-u)\right|_{u=0}=P_{0 L} L^{\prime}(0) P_{0 L}, \\
& \left.\bar{L}_{0 L}^{\prime \prime}(0) \equiv \frac{d^{2}}{d u^{2}} L_{0 L}^{-1}(-u)\right|_{u=0} \neq P_{0 L} L^{\prime \prime}(0) P_{0 L}, \\
& \left.\bar{L}_{0 L}^{\prime \prime \prime}(0) \equiv \frac{d^{3}}{d u^{3}} L_{0 L}^{-1}(-u)\right|_{u=0} \neq P_{0 L} L^{\prime \prime}(0) P_{0 L}, \\
& f=6\left[\operatorname{Tr}\left(K_{+}^{(2)}(0) L_{0 L}^{\prime \prime}(0) P_{0 L}\right)+\operatorname{Tr}\left(K_{+}^{(2)}(0) P_{L 0} \bar{L}_{0 L}^{\prime \prime}(0)\right)+\operatorname{Tr} K_{+}^{(2) \prime \prime}(0)\right] .
\end{aligned}
$$

Using above formulae and after lengthy and tough calculation, one can derive the boundary terms presented by Eqs. (2.3) and (2.4). It was seen that the Hamiltonian of the model can be derived from expansion of the boundary transfer matrix (2.11) at $u=0$ up to the fourth, third and second orders, respectively. This contrasts to the case of two coupled $X Y$ spin chain where the Hamiltonian with four classes of the boundary terms coming from the third and second derivatives of the transfer matrix [26]. In the next sections, we shall be focusing on the solution of the eigenvalue problem of the Hamiltonian (2.1).

\section{The Bethe ansatz equations}

Having established the quantum integrability of the model, let us now solve it by using the coordinate space Bethe ansatz method. Following [19], we assume that the eigenfunction of the Hamiltonian (2.1) takes the form

$$
\begin{aligned}
& |\Psi\rangle=\sum_{\left\{\left(x_{j}, \alpha_{j}\right)\right\}} \Psi_{\alpha_{1}, \ldots, \alpha_{N}} \sigma_{x_{1} \alpha_{1}}^{+} \ldots \sigma_{x_{N} \alpha_{N}}^{+}|0\rangle, \\
& \Psi_{\alpha_{1}, \ldots, \alpha_{N}}\left(x_{1}, \ldots, x_{N}\right) \\
& \quad=\sum_{P} \epsilon_{P} A_{\alpha_{Q 1}, \ldots, \alpha_{Q N}}\left(k_{P Q 1}, \ldots, k_{P Q N}\right) \exp \left(i \sum_{j=1}^{N} k_{P_{j}} x_{j}\right),
\end{aligned}
$$

where the summation is taken over all permutations and negations of $k_{1}, \ldots, k_{N}$, and $Q$ is the permutation of the $N$ particles such that $1 \leqslant x_{Q 1} \leqslant \cdots \leqslant x_{Q N} \leqslant L$. The symbol $\epsilon_{P}$ is a sign factor \pm 1 and changes its sign under each 'mutation'. Substituting the wavefunction into the eigenvalue equation $H|\Psi\rangle=E|\Psi\rangle$, one gets

$$
\begin{aligned}
& A_{\ldots, \alpha_{j}, \alpha_{i}, \ldots}\left(\ldots, k_{j}, k_{i}, \ldots\right)=S_{i j}\left(k_{i}, k_{j}\right) A_{\ldots, \alpha_{i}, \alpha_{j}, \ldots}\left(\ldots, k_{i}, k_{j}, \ldots\right), \\
& A_{\alpha_{i}, \ldots}\left(-k_{j}, \ldots\right)=s^{L}\left(k_{j} ; p_{1 \alpha_{i}}\right) A_{\alpha_{i}, \ldots}\left(k_{j}, \ldots\right), \\
& A_{\ldots, \alpha_{i}}\left(\ldots,-k_{j}\right)=s^{R}\left(k_{j} ; p_{L \alpha_{i}}\right) A_{\ldots, \alpha_{i}}\left(\ldots, k_{j}\right)
\end{aligned}
$$


with $S_{i j}\left(k_{i}, k_{j}\right) \equiv S_{i j}\left(\frac{1}{2}\left(k_{i}-k_{j}\right)\right)$ being the two-particle scattering matrix,

$$
\begin{aligned}
& S_{\alpha \alpha}^{\alpha \alpha}(k)=1, \quad S_{\alpha \beta}^{\alpha \beta}(k)=\frac{\sin k}{\sin (k-i \eta)}, \\
& S_{\alpha \beta}^{\beta \alpha}(k)=-i e^{i \operatorname{sgn}(\beta-\alpha) k} \frac{\sinh \eta}{\sin (k-i \eta)}, \quad \alpha \neq \beta,
\end{aligned}
$$

and $s^{L}\left(k_{j} ; p_{1 \alpha_{i}}\right)$ and $s^{R}\left(k_{j} ; p_{L \alpha_{i}}\right)$ the boundary scattering matrices,

$$
s^{L}\left(k_{j} ; p_{1 \alpha_{i}}\right)=\frac{1-p_{1 \alpha_{i}} e^{i k_{j}}}{1-p_{1 \alpha_{i}} e^{-i k_{j}}}, \quad s^{R}\left(k_{j} ; p_{L \alpha_{i}}\right)=\frac{1-p_{L \alpha_{i}} e^{-i k_{j}}}{1-p_{L \alpha_{i}} e^{i k_{j}}} e^{2 i k_{j}(L+1)},
$$

where $\alpha_{i}=1,2,3$ and $p_{1 \alpha_{i}}$ and $p_{L \alpha_{i}}$ are given by the following formulae, corresponding to the nine cases with respect to the boundary pair $\left(B_{1}^{(m)}, B_{L}^{(l)}\right)$, respectively,

$$
\text { Case (i): } \quad \begin{aligned}
p_{11} & =p_{12}=p_{13} \equiv p_{1}=\frac{1}{c_{-} \exp (4 \eta)}, \\
p_{L 1} & =p_{L 2}=p_{L 3} \equiv p_{L}=\frac{1}{c_{+} \exp (2 \eta)}
\end{aligned}
$$

Case (ii): $\quad p_{11}=0, \quad p_{12}=p_{13} \equiv p_{1+}=\frac{1}{c_{-}}$,

$$
p_{L 1}=p_{L 2} \equiv p_{L+}=\frac{1}{c_{+} \exp (2 \eta)}, \quad p_{L 3}=0
$$

Case (iii): $\quad p_{11}=p_{12}=0, \quad p_{13}=\frac{1}{c_{-}}$,

$$
p_{L 1}=\frac{1}{c_{+}}, \quad p_{L 2}=p_{L 3}=0
$$

Case (iv): $\quad p_{11}=p_{12}=p_{13} \equiv p_{1}=\frac{1}{c_{-} \exp (4 \eta)}$,

$$
p_{L 1}=p_{L 2}=\frac{1}{c_{+} \exp (2 \eta)}, \quad p_{L 3}=0
$$

Case (v): $\quad p_{11}=p_{12}=p_{13} \equiv p_{1}=\frac{1}{c_{-} \exp (4 \eta)}$,

$$
p_{L 1}=\frac{1}{c_{+}}, \quad p_{L 2}=p_{L 3}=0
$$

Case (vi): $\quad p_{11}=0, \quad p_{12}=p_{13}=\frac{1}{c_{-}}$,

$$
p_{L 1}=\frac{1}{c_{+}}, \quad p_{L 2}=p_{L 3}=0
$$

Case (vii): $\quad p_{11}=0, \quad p_{12}=p_{13}=\frac{1}{c_{-}}$,

$$
p_{L 1}=p_{L 2}=p_{L 3} \equiv p_{L}=\frac{1}{c_{+} \exp (2 \eta)}
$$


Case (viii): $\quad p_{11}=p_{12}=0, \quad p_{13}=\frac{1}{c_{-}}$,

$$
p_{L 1}=p_{L 2}=p_{L 3} \equiv p_{L}=\frac{1}{c_{+} \exp (2 \eta)}
$$

Case (ix): $\quad p_{11}=p_{12}=0, \quad p_{13}=\frac{1}{c_{-}}$,

$$
p_{L 1}=p_{L 2}=\frac{1}{c_{+} \exp (2 \eta)}, \quad p_{L 3}=0
$$

As is seen above, the two-particle $S$-matrix (3.4) is nothing but the $R$ matrix of the $A_{2}$ $X X Z$ Heisenberg model (in the homogeneous gauge) and thus satisfies the quantum YangBaxter equation, and the boundary scattering matrices $s^{L}$ and $s^{R}$ obey the corresponding reflection equations (for details, see, [29]). This is seen as follows. One introduces the notation

$$
s(k ; p)=\frac{1-p e^{-i k}}{1-p e^{i k}} .
$$

Then the boundary scattering matrices $s^{L}\left(k_{j} ; p_{1 \alpha_{i}}\right), s^{R}\left(k_{j} ; p_{L \alpha_{i}}\right)$ can be written as, corresponding to the nine cases, respectively,

Case (i): $\quad s^{L}\left(k_{j} ; p_{1 \alpha_{i}}\right)=s\left(-k_{j} ; p_{1}\right) I$,

$$
s^{R}\left(k_{j} ; p_{L \alpha_{i}}\right)=e^{i k_{j} 2(L+1)} s\left(k_{j} ; p_{L}\right) I
$$

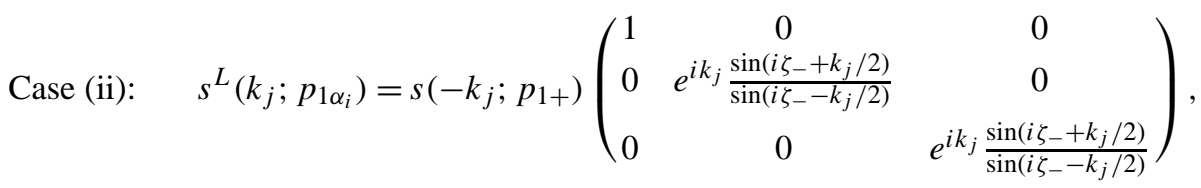

$$
s^{R}\left(k_{j} ; p_{L \alpha_{i}}\right)=e^{i k_{j} 2(L+1)} s\left(k_{j} ; p_{L 1}\right)\left(\begin{array}{ccc}
1 & 0 & 0 \\
0 & 1 & 0 \\
0 & 0 & e^{i k_{j}} \frac{\sin \left(i \kappa_{+}+k_{j} / 2\right)}{\sin \left(i \kappa_{+}-k_{j} / 2\right)}
\end{array}\right)
$$

Case (iii): $\quad s^{L}\left(k_{j} ; p_{1 \alpha_{i}}\right)=s\left(-k_{j} ; p_{11}\right)\left(\begin{array}{ccc}1 & 0 & 0 \\ 0 & 1 & 0 \\ 0 & 0 & e^{i k_{j}} \frac{\sin \left(i \kappa_{-}+k_{j} / 2\right)}{\sin \left(i \kappa_{-}-k_{j} / 2\right)}\end{array}\right)$,

$$
\begin{aligned}
s^{R}\left(k_{j} ; p_{L \alpha_{i}}\right)= & e^{i k_{j} 2(L+1)} s\left(k_{j} ; p_{L 1}\right) \\
& \times\left(\begin{array}{ccc}
1 & 0 & 0 \\
0 & e^{i k_{j} \frac{\sin \left(i \zeta_{+}+k_{j} / 2\right)}{\sin \left(i \zeta_{+}-k_{j} / 2\right)}} & 0 \\
0 & 0 & e^{i k_{j}} \frac{\sin \left(i \zeta_{+}+k_{j} / 2\right)}{\sin \left(i \zeta_{+}-k_{j} / 2\right)}
\end{array}\right) ;
\end{aligned}
$$

Case (iv): $\quad s^{L}\left(k_{j} ; p_{1 \alpha_{i}}\right)=s\left(-k_{j} ; p_{1}\right) I$,

$$
s^{R}\left(k_{j} ; p_{L \alpha_{i}}\right)=e^{i k_{j} 2(L+1)} s\left(k_{j} ; p_{L 1}\right)\left(\begin{array}{ccc}
1 & 0 & 0 \\
0 & 1 & 0 \\
0 & 0 & e^{i k_{j}} \frac{\sin \left(i \kappa_{+}+k_{j} / 2\right)}{\sin \left(i \kappa_{+}-k_{j} / 2\right)}
\end{array}\right)
$$


Case (v): $\quad s^{L}\left(k_{j} ; p_{1 \alpha_{i}}\right)=s\left(-k_{j} ; p_{1}\right) I$,

$$
\begin{aligned}
s^{R}\left(k_{j} ; p_{L \alpha_{i}}\right)= & e^{i k_{j} 2(L+1)} s\left(k_{j} ; p_{L 1}\right) \\
& \times\left(\begin{array}{ccc}
1 & 0 & 0 \\
0 & e^{i k_{j} \frac{\sin \left(i \zeta_{+}+k_{j} / 2\right)}{\sin \left(i \zeta_{+}-k_{j} / 2\right)}} & 0 \\
0 & 0 & e^{i k_{j}} \frac{\sin \left(i \zeta_{+}+k_{j} / 2\right)}{\sin \left(i \zeta_{+}-k_{j} / 2\right)}
\end{array}\right) ;
\end{aligned}
$$

Case (vi): $\quad s^{L}\left(k_{j} ; p_{1 \alpha_{i}}\right)=s\left(-k_{j} ; p_{1+}\right)\left(\begin{array}{ccc}1 & 0 & 0 \\ 0 & e^{i k_{j} \frac{\sin \left(i \zeta_{-}+k_{j} / 2\right)}{\sin \left(i \zeta_{-}-k_{j} / 2\right)}} & 0 \\ 0 & 0 & e^{i k_{j} \frac{\sin \left(i \zeta_{-}+k_{j} / 2\right)}{\sin \left(i \zeta_{-}-k_{j} / 2\right)}}\end{array}\right)$,

$$
s^{R}\left(k_{j} ; p_{L \alpha_{i}}\right)=e^{i k_{j} 2(L+1)} s\left(k_{j} ; p_{L 1}\right)
$$

$$
\times\left(\begin{array}{ccc}
1 & 0 & 0 \\
0 & e^{i k_{j} \frac{\sin \left(i \zeta_{+}+k_{j} / 2\right)}{\sin \left(i \zeta_{+}-k_{j} / 2\right)}} & 0 \\
0 & 0 & e^{i k_{j}} \frac{\sin \left(i \zeta_{+}+k_{j} / 2\right)}{\sin \left(i \zeta_{+}-k_{j} / 2\right)}
\end{array}\right)
$$

Case (vii): $\quad s^{L}\left(k_{j} ; p_{1 \alpha_{i}}\right)=s\left(-k_{j} ; p_{1+}\right)\left(\begin{array}{ccc}1 & 0 & 0 \\ 0 & e^{i k_{j} \frac{\sin \left(i \zeta_{-}+k_{j} / 2\right)}{\sin \left(i \zeta_{-}-k_{j} / 2\right)}} & 0 \\ 0 & 0 & e^{i k_{j} \frac{\sin \left(i \zeta_{-}+k_{j} / 2\right)}{\sin \left(i \zeta_{-}-k_{j} / 2\right)}}\end{array}\right)$,

$$
s^{R}\left(k_{j} ; p_{L \alpha_{i}}\right)=e^{i k_{j} 2(L+1)} s\left(k_{j} ; p_{L}\right) I
$$

Case (viii): $\quad s^{L}\left(k_{j} ; p_{1 \alpha_{i}}\right)=s\left(-k_{j} ; p_{11}\right)\left(\begin{array}{ccc}1 & 0 & 0 \\ 0 & 1 & 0 \\ 0 & 0 & e^{i k_{j}} \frac{\sin \left(i \kappa_{-}+k_{j} / 2\right)}{\sin \left(i \kappa_{-}-k_{j} / 2\right)}\end{array}\right)$,

$$
s^{R}\left(k_{j} ; p_{L \alpha_{i}}\right)=e^{i k_{j} 2(L+1)} s\left(k_{j} ; p_{L}\right) I
$$

Case (ix): $\quad s^{L}\left(k_{j} ; p_{1 \alpha_{i}}\right)=s\left(-k_{j} ; p_{11}\right)\left(\begin{array}{ccc}1 & 0 & 0 \\ 0 & 1 & 0 \\ 0 & 0 & e^{i k_{j}} \frac{\sin \left(i \kappa_{-}+k_{j} / 2\right)}{\sin \left(i \kappa_{-}-k_{j} / 2\right)}\end{array}\right)$,

$$
s^{R}\left(k_{j} ; p_{L \alpha_{i}}\right)=e^{i k_{j} 2(L+1)} s\left(k_{j} ; p_{L 1}\right)\left(\begin{array}{ccc}
1 & 0 & 0 \\
0 & 1 & 0 \\
0 & 0 & e^{i k_{j}} \frac{\sin \left(i \kappa_{+}+k_{j} / 2\right)}{\sin \left(i \kappa_{+}-k_{j} / 2\right)}
\end{array}\right) .
$$

Here $I$ stands for $3 \times 3$ identity matrix and $p_{1+}, p_{L+}$ are the ones given in (3.6); $\zeta_{ \pm}, \kappa_{ \pm}$ are parameters defined by

$$
e^{2 \zeta_{ \pm}}=c_{ \pm}, \quad e^{2 \kappa_{+}}=c_{+} e^{2 \eta}, \quad e^{2 \kappa_{-}}=c_{-} .
$$

We immediately see that (3.15) are the trivial solutions of the reflection equations, whereas (3.16) and (3.17) are the diagonal solutions for the $A_{2} X X Z$ model $R$-matrix. The boundary scattering matrices given in (3.15), (3.16) and (3.17) also constitute nine classes of possible choices of boundary conditions for the spin degrees of freedom of the model. 
Then, the diagonalization of Hamiltonian (2.1) reduces to solving the following matrix eigenvalue equation

$$
T_{j} t=t, \quad j=1, \ldots, N
$$

where $t$ denotes an eigenvector on the space of the spin variables and $T_{j}$ takes the form

$$
T_{j}=S_{j}^{-}\left(k_{j}\right) s^{L}\left(-k_{j} ; p_{1 \alpha_{j}}\right) R_{j}^{-}\left(k_{j}\right) R_{j}^{+}\left(k_{j}\right) s^{R}\left(k_{j} ; p_{L \alpha_{j}}\right) S_{j}^{+}\left(k_{j}\right)
$$

with

$$
\begin{aligned}
S_{j}^{+}\left(k_{j}\right) & =S_{j, N}\left(k_{j}, k_{N}\right) \ldots S_{j, j+1}\left(k_{j}, k_{j+1}\right), \\
S_{j}^{-}\left(k_{j}\right) & =S_{j, j-1}\left(k_{j}, k_{j-1}\right) \ldots S_{j, 1}\left(k_{j}, k_{1}\right), \\
R_{j}^{-}\left(k_{j}\right) & =S_{1, j}\left(k_{1},-k_{j}\right) \ldots S_{j-1, j}\left(k_{j-1},-k_{j}\right), \\
R_{j}^{+}\left(k_{j}\right) & =S_{j+1, j}\left(k_{j+1},-k_{j}\right) \ldots S_{N, j}\left(k_{N},-k_{j}\right) .
\end{aligned}
$$

This problem may be solved using the algebraic Bethe ansatz method. The Bethe ansatz equations are

$$
\begin{aligned}
& e^{i k_{j} 2(L+1)} F\left(k_{j} ; p_{11}, p_{L 1}\right)=\prod_{\alpha=1}^{M_{1}} \frac{\sin \left[\frac{1}{2}\left(k_{j}-\Lambda_{\alpha}^{(1)}\right)+\frac{i \eta}{2}\right]}{\sin \left[\frac{1}{2}\left(k_{j}-\Lambda_{\alpha}^{(1)}\right)-\frac{i \eta}{2}\right]} \frac{\sin \left[\frac{1}{2}\left(k_{j}+\Lambda_{\alpha}^{(1)}\right)+\frac{i \eta}{2}\right]}{\sin \left[\frac{1}{2}\left(k_{j}+\Lambda_{\alpha}^{(1)}\right)-\frac{i \eta}{2}\right]}, \\
& \prod_{\alpha=1}^{N} \frac{\sin \left[\frac{1}{2}\left(\Lambda_{\gamma}^{(1)}-k_{\alpha}\right)+\frac{i \eta}{2}\right]}{\sin \left[\frac{1}{2}\left(\Lambda_{\gamma}^{(1)}-k_{\alpha}\right)-\frac{i \eta}{2}\right]} \frac{\sin \left[\frac{1}{2}\left(\Lambda_{\gamma}^{(1)}+k_{\alpha}+\frac{i \eta}{2}\right]\right.}{\sin \left[\frac{1}{2}\left(\Lambda_{\gamma}^{(1)}+k_{\alpha}\right)-\frac{i \eta}{2}\right]} \\
& =G\left(\Lambda_{\gamma}^{(1)} ; \zeta_{-}, \zeta_{+}\right) \prod_{\substack{\gamma^{\prime}=1 \\
\gamma^{\prime} \neq \gamma}}^{M_{1}} \frac{\sin \left[\frac{1}{2}\left(\Lambda_{\gamma}^{(1)}-\Lambda_{\gamma^{\prime}}^{(1)}\right)+i \eta\right]}{\sin \left[\frac{1}{2}\left(\Lambda_{\gamma}^{(1)}-\Lambda_{\gamma^{\prime}}^{(1)}\right)-i \eta\right]} \frac{\sin \left[\frac{1}{2}\left(\Lambda_{\gamma}^{(1)}+\Lambda_{\gamma^{\prime}}^{(1)}\right)+i \eta\right]}{\sin \left[\frac{1}{2}\left(\Lambda_{\gamma}^{(1)}+\Lambda_{\gamma^{\prime}}^{(1)}\right)-i \eta\right]} \\
& \times \prod_{\delta=1}^{M_{2}} \frac{\sin \left[\frac{1}{2}\left(\Lambda_{\gamma}^{(1)}-\lambda_{\delta}^{(2)}\right)-\frac{i \eta}{2}\right]}{\sin \left[\frac{1}{2}\left(\Lambda_{\gamma}^{(1)}-\lambda_{\delta}^{(2)}\right)+\frac{i \eta}{2}\right]} \frac{\sin \left[\frac{1}{2}\left(\Lambda_{\gamma}^{(1)}+\lambda_{\delta}^{(2)}\right)-\frac{i \eta}{2}\right]}{\sin \left[\frac{1}{2}\left(\Lambda_{\gamma}^{(1)}+\lambda_{\delta}^{(2)}\right)+\frac{i \eta}{2}\right]}, \\
& \prod_{\substack{\gamma^{\prime}=1 \\
\gamma^{\prime} \neq \gamma}}^{M_{2}} \frac{\sin \left[\frac{1}{2}\left(\Lambda_{\gamma}^{(2)}-\Lambda_{\gamma^{\prime}}^{(2)}\right)+i \eta\right]}{\sin \left[\frac{1}{2}\left(\Lambda_{\gamma}^{(2)}-\Lambda_{\gamma^{\prime}}^{(2)}\right)-i \eta\right]} \frac{\sin \left[\frac{1}{2}\left(\Lambda_{\gamma}^{(2)}+\Lambda_{\gamma^{\prime}}^{(2)}\right)+i \eta\right]}{\sin \left[\frac{1}{2}\left(\Lambda_{\gamma}^{(2)}+\Lambda_{\gamma^{\prime}}^{(2)}\right)-i \eta\right]}=K\left(\Lambda_{\gamma}^{(2)} ; \kappa_{-}, \kappa_{+}\right) \\
& \times \prod_{\alpha=1}^{M_{1}} \frac{\sin \left[\frac{1}{2}\left(\Lambda_{\gamma}^{(2)}-\Lambda_{\alpha}^{(1)}\right)+\frac{i \eta}{2}\right]}{\sin \left[\frac{1}{2}\left(\Lambda_{\gamma}^{(2)}-\Lambda_{\alpha}^{(1)}\right)-\frac{i \eta}{2}\right]} \frac{\sin \left[\frac{1}{2}\left(\Lambda_{\gamma}^{(2)}+\Lambda_{\alpha}^{(1)}\right)+\frac{i \eta}{2}\right]}{\sin \left[\frac{1}{2}\left(\Lambda_{\gamma}^{(2)}+\Lambda_{\alpha}^{(1)}\right)-\frac{i \eta}{2}\right]}
\end{aligned}
$$

where

$F\left(k_{j} ; p_{1+}, p_{L+}\right)=s\left(k_{j} ; p_{1+}\right) s\left(k_{j} ; p_{L+}\right) \quad($ for all cases $)$, 


$$
\begin{aligned}
& G\left(\Lambda_{\gamma}^{(1)} ; \zeta_{-}, \zeta_{+}\right)= \begin{cases}1, & \text { case (i), } \\
\frac{\sin \left(i \zeta_{-}-i \Lambda^{(1)}-\gamma / 2+\eta / 2\right)}{\sin \left(i \zeta_{-}+i \Lambda^{(1)}-\gamma / 2+\eta / 2\right)} e^{i \Lambda_{\gamma}^{(1)},} & \text { case (ii), } \\
\frac{\sin \left(i \zeta_{+}-i \Lambda^{(1)}-\gamma / 2+\eta / 2\right)}{\sin \left(i \zeta_{+}+i \Lambda^{(1)}-\gamma / 2+\eta / 2\right)} e^{-i \Lambda_{\gamma}^{(1)},} & \text { case (iii), } \\
1, & \text { case (iv), } \\
\frac{\sin \left(i \zeta_{+}-i \Lambda^{(1)}-\gamma / 2+\eta / 2\right)}{\sin \left(i \zeta_{+}+i \Lambda^{(1)}-\gamma / 2+\eta / 2\right)} e^{-i \Lambda_{\gamma}^{(1)},} & \text { case (v), } \\
\frac{\sin \left(i \zeta_{-}-i \Lambda^{(1)}-\gamma / 2+\eta / 2\right)}{\sin \left(i \zeta_{-}+i \Lambda^{(1)}-\gamma / 2+\eta / 2\right)} \frac{\sin \left(i \zeta_{+}-i \Lambda^{(1)}-\gamma / 2+\eta / 2\right)}{\sin \left(i \zeta_{+}+i \Lambda^{(1)}-\gamma / 2+\eta / 2\right)}, & \text { case (vi), } \\
\frac{\sin \left(i \zeta_{-}-i \Lambda^{(1)}-\gamma / 2+\eta / 2\right)}{\sin \left(i \zeta_{-}+i \Lambda^{(1)}-\gamma / 2+\eta / 2\right)} e^{i \Lambda_{\gamma}^{(1)},} & \text { case (vii), } \\
1, & \text { case (viii), } \\
1, & \text { case (ix), }\end{cases} \\
& K\left(\Lambda_{\gamma}^{(2)} ; \kappa_{-}, \kappa_{+}\right)= \begin{cases}1, & \text { case (i), } \\
\frac{\sin \left(i \kappa_{+}-i \Lambda^{(2)}-\gamma / 2+\eta\right)}{\sin \left(i \kappa_{+}+i \Lambda^{(2)}-\gamma / 2+\eta\right)} e^{-i \Lambda_{\gamma}^{(2)},} & \text { case (ii), } \\
\frac{\sin \left(i \kappa_{-}-i \Lambda^{(2)}-\gamma / 2+\eta\right)}{\sin \left(i \kappa_{-}+i \Lambda^{(2)}-\gamma / 2+\eta\right)} e^{i \Lambda_{\gamma}^{(2)}}, & \text { case (iii), } \\
\frac{\sin \left(i \kappa_{+}-i \Lambda^{(2)}-\gamma / 2+\eta\right)}{\sin \left(i \kappa_{+}+i \Lambda^{(2)}-\gamma / 2+\eta\right)} e^{-i \Lambda_{\gamma}^{(2)},} & \text { case (iv), } \\
1, & \text { case (v), } \\
1, & \text { case (vi), } \\
1, & \text { case (vii), } \\
\frac{\sin \left(i \kappa_{-}-i \Lambda^{(2)}-\gamma / 2+\eta\right)}{\sin \left(i \kappa_{-}+i \Lambda^{(2)}-\gamma / 2+\eta\right)} e^{i \Lambda_{\gamma}^{(2)},} & \text { case (viii), } \\
\frac{\sin \left(i \kappa_{-}-i \Lambda^{(2)}-\gamma / 2+\eta\right)}{\sin \left(i \kappa_{-}+i \Lambda^{(2)}-\gamma / 2+\eta\right)} \frac{\sin \left(i \kappa_{+}-i \Lambda^{(2)}-\gamma / 2+\eta\right)}{\sin \left(i \kappa_{+}+i \Lambda^{(2)}-\gamma / 2+\eta\right)}, & \text { case (ix). }\end{cases}
\end{aligned}
$$

The energy eigenvalue $E$ of the model is given by $E=-2 \sum_{j=1}^{N} \cos k_{j}$ (modular an unimportant additive constant).

\section{Conclusion}

In this paper, we have studied integrable open-boundary conditions for the three coupled $X Y$ spin chain model. The quantum integrability of the boundary system has been established by the fact that the corresponding Hamiltonian may be embedded into a one-parameter family of commuting transfer matrices. A desirable way to handle open boundary conditions for the models associated with a general class of quantum $R$-matrices (with or without crossing-unitarity) has been developed. Moreover, the Bethe ansatz equations are derived by means of the coordinate Bethe ansatz approach. This provides a basis for computing the finite size corrections to the low-lying energies in the system, which in turn could be used together with the boundary conformal field theory technique to study the critical properties of the boundaries.

Lastly, it is interesting to formulate a graded version of the quantum Yang-Baxter algebra and reflection equation algebra for the fermionic Bariev model. The algebraic Bethe ansatz for the three coupled $X Y$ model with both periodic and open boundary 
conditions would be very significant in understanding of the symmetry structure of the model. Those will be addressed in a future publication.

\section{Acknowledgements}

A.F., I.R. and X.W.G. thank CNPq (Conselho Nacional de Desenvolvimento Científico e Tecnológico) and FAPERGS (Fundação de Amparo ã Pesquisa do Estado do Rio Grande do Sul) for financial support, I.R. also thanks PRONEX. M.D.G. and H.Q.Z. acknowledges the support from the Australian Research Council. We thank R. Mckenzie and Jon Links for helpful comments on the manuscript.

\section{Appendix A. Derivation of the boundary $K_{ \pm}$-matrices}

The $R$-matrix is a $64 \times 64$ matrix with 216 nonzero elements which are given in [27]. We now are looking for diagonal solutions $K_{ \pm}(u)$ of the REs. We parametrize $K_{ \pm}(u)$ as

$$
K_{-}(u)=\left(\begin{array}{cccccccc}
z 1_{ \pm}(u) & 0 & 0 & 0 & 0 & 0 & 0 & 0 \\
0 & z 2_{ \pm}(u) & 0 & 0 & 0 & 0 & 0 & 0 \\
0 & 0 & z \pm 3(u) & 0 & 0 & 0 & 0 & 0 \\
0 & 0 & 0 & z 4_{ \pm}(u) & 0 & 0 & 0 & 0 \\
0 & 0 & 0 & 0 & z 5_{ \pm}(u) & 0 & 0 & 0 \\
0 & 0 & 0 & 0 & 0 & z 6_{ \pm}(u) & 0 & 0 \\
0 & 0 & 0 & 0 & 0 & 0 & z 7_{ \pm}(u) & 0 \\
0 & 0 & 0 & 0 & 0 & 0 & 0 & z 8_{ \pm}(u)
\end{array}\right)
$$

Using some simpler functional equations from the first RE wich are given by (A.1)-(A.8) in the Appendix A of the paper [28], one can present an ansatz

$$
\begin{array}{lll}
\frac{z 2_{-}(u)}{z 1_{-}(u)}=\frac{c_{1}-c_{8} u}{c_{1}+c_{8} u}, & \frac{z 3_{-}(u)}{z 1_{-}(u)}=\frac{c_{2}-c_{9} u}{c_{2}+c_{9} u}, \\
\frac{z 5_{-}(u)}{z 1_{-}(u)}=\frac{c_{3}-c_{10} u}{c_{3}+c_{10} u}, & \frac{z 4_{-}(u)}{z 2_{-}(u)}=\frac{c_{4}-c_{11} u}{c_{4}+c_{11} u}, \\
\frac{z 6_{-}(u)}{z 2_{-}(u)}=\frac{c_{5}-c_{12} u}{c_{5}+c_{12} u}, & \frac{z 7_{-}(u)}{z 3_{-}(u)}=\frac{c_{6}-c_{13} u}{c_{6}+c_{13} u}, \\
\frac{z 8_{-}(u)}{z 4_{-}(u)}=\frac{c_{7}-c_{14} u}{c_{7}+c_{14} u}, &
\end{array}
$$

with minimal coefficients $c_{i}$ to be determined. Running the RE again with above ansatz, it can be found that only one coefficient is free and three classes of boundary $K_{-}$-matrices can be immediately chosen as the forms presented in (2.17).

To solve the second RE is rather cumbersome and sophisticated. For clarifying the functional equations arising from the second RE, we denote the Boltzmann weights associated with the $R$-matrix as $w_{1}(u, v), \ldots, w_{58}(u, v)$ in accordance with the orders listed in the paper [27]. The convenient notations $\hat{z}=z_{+}(v)$ and $\bar{z}=z_{+}(u)$ will be implied 
hereafter. Similarly, after substituting $K_{+}$-matrix (A.1) into the RE (2.13), we may pick up some simpler functional equations such as

$$
\begin{aligned}
& \frac{\hat{z} 2}{\hat{z} 1}=\frac{w_{2}(v, u) \tilde{\rho}_{1}(v,-u) \bar{z} 2+w_{3}(v, u) \tilde{\rho}_{2}(v,-u) \bar{z} 1}{w_{3}(-u,-v) \tilde{\rho}_{2}(u,-v) e^{-4 \eta} \bar{z} 2+w_{2}(-u,-v) \tilde{\rho}_{1}(u,-v) \bar{z} 1}, \\
& \frac{\hat{z} 3}{\hat{z} 1}=\frac{w_{2}(v, u) \tilde{\rho}_{1}(v,-u) e^{2 \eta} \bar{z} 3+w_{3}(v, u) \tilde{\rho}_{2}(v,-u) \bar{z} 1}{w_{3}(-u,-v) \tilde{\rho}_{2}(u,-v) \bar{z} 3+w_{2}(-u,-v) \tilde{\rho}_{1}(u,-v) e^{2 \eta} \bar{z} 1}, \\
& \frac{\hat{z} 5}{\hat{z} 1}=\frac{w_{2}(v, u) \tilde{\rho}_{1}(v,-u) \bar{z} 5+w_{3}(v, u) \tilde{\rho}_{2}(v,-u) e^{-4 \eta} \bar{z} 1}{w_{3}(-u,-v) \tilde{\rho}_{2}(u,-v) \bar{z} 5+w_{2}(-u,-v) \tilde{\rho}_{1}(u,-v) \bar{z} 1}, \\
& \frac{\hat{z} 8}{\hat{z} 6}=\frac{w_{47}(-u,-v) \tilde{\rho}_{3}(u,-v) e^{2 \eta} \bar{z} 6+w_{46}(-u,-v) \tilde{\rho}_{4}(u,-v) \bar{z} 8}{w_{46}(v, u) \tilde{\rho}_{4}(v,-u) \bar{z} 6+w_{47}(v, u) \tilde{\rho}_{3}(v,-u) e^{2 \eta} \bar{z} 8}, \\
& \frac{\hat{z} 6}{\hat{z} 2}=\frac{w_{19}(-u,-v) \tilde{\rho}_{6}(u,-v) e^{2 \eta} \bar{z} 2+w_{20}(-u,-v) \tilde{\rho}_{5}(u,-v) e^{4 \eta} \bar{z} 6}{w_{19}(v, u) \tilde{\rho}_{6}(v,-u) e^{2 \eta} \bar{z} 6+w_{20}(v, u) \tilde{\rho}_{5}(v,-u) \bar{z} 2}, \\
& \frac{\hat{z} 4}{\hat{z} 2}=\frac{w_{20}(-u,-v) \tilde{\rho}_{5}(u,-v) \bar{z} 2+w_{19}(-u,-v) \tilde{\rho}_{7}(u,-v) \bar{z} 4}{w_{19}(v, u) \tilde{\rho}_{7}(v,-u) \bar{z} 2+w_{20}(v, u) \tilde{\rho}_{5}(v,-u) \bar{z} 4}, \\
& \frac{\hat{z} 7}{\hat{z} 3}=\frac{w_{19}(-u,-v) \tilde{\rho}_{7}(u,-v) e^{2 \eta} \bar{z} 7+w_{20}(-u,-v) \tilde{\rho}_{5}(u,-v) \bar{z} 3}{w_{19}(v, u) \tilde{\rho}_{7}(v,-u) e^{2 \eta} \bar{z} 3+w_{20}(v, u) \tilde{\rho}_{5}(v,-u) e^{4 \eta} \bar{z} 7} .
\end{aligned}
$$

Before further going on, we first need to work out the matrices $R_{21}^{\mathrm{t}_{1} \mathrm{t}_{2}}(u, v), R_{12}^{\mathrm{t}_{1} \mathrm{t}_{2}}(u, v)$, $\widetilde{R}_{12}(u, v)$ and $\widetilde{R}_{21}(u, v)$ according to their definitions in Section 2. For our convenience, we prefer to present below some entries of $\widetilde{R}_{12}$ involving in above equations as

$$
\begin{aligned}
& \tilde{\rho}_{1}(u, v)=-\frac{\left(1+e^{6 \eta} u v\right)\left(1+e^{4 \eta} u v\right)^{2}\left(1+e^{2 \eta} u v\right)(1+u v)}{(u-v)^{2}\left(e^{2 \eta} u-v\right)\left(e^{2 \eta} v-u\right)\left(e^{4 \eta} u-v\right) e^{4 \eta}} \\
& \tilde{\rho}_{2}(u, v)=\frac{\left(1+e^{6 \eta} u v\right)\left(1+e^{4 \eta} u v\right)^{2}\left(1+e^{2 \eta} u v\right)(1+u v) \sqrt{\left(1+e^{4 \eta} u^{2}\right)\left(1+e^{4 \eta} v^{2}\right)}}{(u-v)^{2}\left(e^{2 \eta} u-v\right)\left(e^{2 \eta} v-u\right)\left(e^{4 \eta} u-v\right)\left(e^{4 \eta} v-u\right) e^{2 \eta}} \\
& \tilde{\rho}_{3}(u, v)=\frac{\left(1+e^{4 \eta} u v\right)\left(1+e^{2 \eta} u v\right)\left(u v+e^{2 \eta}\right)(1+u v)^{2} \sqrt{\left(1+u^{2}\right)\left(1+v^{2}\right)}}{(u-v)^{2}\left(e^{2 \eta} u-v\right)\left(e^{2 \eta} v-u\right)\left(e^{4 \eta} u-v\right)\left(e^{4 \eta} v-u\right)} \\
& \tilde{\rho}_{4}(u, v)=\frac{\left(1+e^{4 \eta} u v\right)\left(1+e^{2 \eta} u v\right)\left(u v+e^{2 \eta}\right)(1+u v)^{2}}{(u-v)^{2}\left(e^{2 \eta} u-v\right)\left(e^{2 \eta} v-u\right)\left(e^{4 \eta} v-u\right)} \\
& \tilde{\rho}_{5}(u, v)=\frac{\left(1+e^{4 \eta} u v\right)^{2}\left(1+e^{2 \eta} u v\right)(1+u v)^{2} \sqrt{\left(1+e^{2 \eta} u^{2}\right)\left(1+e^{2 \eta} v^{2}\right)}}{(u-v)^{2}\left(e^{2 \eta} u-v\right)\left(e^{2 \eta} v-u\right)\left(e^{4 \eta} u-v\right)\left(e^{4 \eta} v-u\right)} \\
& \tilde{\rho}_{6}(u, v)=-\frac{\left(1+e^{4 \eta} u v\right)^{2}\left(1+e^{2 \eta} u v\right)(1+u v)^{2}}{(u-v)^{2}\left(e^{2 \eta} u-v\right)\left(e^{2 \eta} v-u\right)\left(e^{4 \eta} u-v\right) e^{\eta}} \\
& \tilde{\rho}_{7}(u, v)=\frac{\left(1+e^{4 \eta} u v\right)^{2}\left(1+e^{2 \eta} u v\right)(1+u v)^{2}}{(u-v)^{2}\left(e^{2 \eta} u-v\right)\left(e^{2 \eta} v-u\right)\left(e^{4 \eta} v-u\right) e^{\eta}} .
\end{aligned}
$$

Then by analyzing the structure of the Eqs. (A.3)-(A.9), we can get the following relations:

$$
\frac{z 2_{+}(u)}{z 1_{+}(u)}=\frac{c_{1} u+e^{4 \eta} c_{8}}{c_{1} u-c_{8}}, \quad \frac{z 3_{+}(u)}{z 1_{+}(u)}=\frac{c_{2} u+e^{2 \eta} c_{9}}{e^{2 \eta} c_{2} u-c_{9}},
$$




$$
\begin{array}{ll}
\frac{z 5_{+}(u)}{z 1_{+}(u)}=\frac{c_{3} u+c_{10}}{e^{4 \eta} c_{3} u-c_{10}}, & \frac{z 4_{+}(u)}{z 2_{+}(u)}=\frac{c_{6} u+e^{2 \eta} c_{11}}{e^{2 \eta} c_{6} u-c_{11}}, \\
\frac{z 6_{+}(u)}{z 2_{+}(u)}=\frac{c_{5} u+c_{12}}{e^{4 \eta} c_{5} u-c_{12}}, & \frac{z 7_{+}(u)}{z 3_{+}(u)}=\frac{c_{7} u+c_{13}}{e^{4 \eta} c_{7} u-c_{13}}, \\
\frac{z 8_{+}(u)}{z 6_{+}(u)}=\frac{c_{4} u+e^{2 \eta} c_{14}}{e^{2 \eta} c_{4} u-c_{14}} . &
\end{array}
$$

Running second RE (2.13) with above relations again and again, the solutions (2.18) would be fixed definitely.

\section{References}

[1] J.B. Bednorz, K.A. Müller, Z. Phys. B 64 (1986) 189.

[2] V.E. Korepin, F.H.L. Essler, Exactly Solvable Models of Strongly Correlated Electrons, World Scientific, Singapore, 1994.

[3] P. Schlottmann, Phys. Rev. Lett. 68 (1992) 1916;

S. Sarkar, J. Phys. A: Math. Gen. 23 (1990) L409;

P.A. Bares, G. Blatter, M. Ogata, Phys. Rev. Lett. 73 (1991) 11340;

I.N. Karnaukhov, Phys. Rev. Lett. 73 (1994) 11340.

[4] F.H.L. Essler, V.E. Korepin, K. Schoutens, Phys. Rev. Lett. 68 (1992) 2960;

F.H.L. Essler, V.E. Korepin, Phys. Rev. B 46 (1992) 9147.

[5] A.J. Bracken, M.D. Gould, J.R. Links, Y.Z. Zhang, Phys. Rev. Lett. 74 (1995) 2768.

[6] E.H. Lieb, F.Y. Wu, Phys. Rev. Lett. 20 (1968) 1445.

[7] P.W. Anderson, Science 235 (1987) 1196;

F.C. Zhang, T.M. Rice, Phys. Rev. B 37 (1988) 3759.

[8] B. Sutherland, Phys. Rev. B 12 (1975) 3795;

P. Schlottmann, Phys. Rev. B 36 (1987) 5177;

P. Wiegmann, Phys. Rev. Lett. 60 (1988) 821;

F.H.L. Essler, V.E. Korepin, Phys. Rev. B 46 (1992) 9147;

A. Foerster, M. Karowski, Phys. Rev. B 46 (1992) 9234;

A. Foerster, M. Karowski, Nucl. Phys. B 396 (1993) 611.

[9] R.Z. Bariev, J. Phys. A: Math. Gen. 24 (1991) L549;

R.Z. Bariev, J. Phys. A: Math. Gen. 24 (1991) L919.

[10] R.Z. Bariev, A. Klümper, A. Schadschneider, J. Zittartz, J. Phys. A: Math. Gen. 26 (1993) 4663;

R.Z. Bariev, A. Klümper, A. Schadschneider, J. Zittartz, J. Phys. A: Math. Gen. 26 (1993) 1249.

[11] E.K. Sklyanin, L.D. Faddeev, Sov. Phys. Dokl. 23 (1978) 902;

E.K. Sklyanin, J. Sov. Math. 19 (1982) 1546.

[12] P.P. Kulish, E.K. Sklyanin, Lecture Notes in Physics, Vol. 151, Springer-Verlag, Berlin, 1982, p. 61.

[13] V.E. Korepin, N.M. Bogoliubov, A.G. Izergin, Quantum Inverse Scattering Method and Correlation Function, Cambridge Univ. Press, 1993.

[14] F.C. Alcaraz, M.N. Barber, M.T. Batchelor, R.J. Baxter, G.R.W. Quispel, J. Phys. A: Math. Gen. 20 (1987) 6397;

I.V. Cherednik, Theor. Math. Phys. 61 (1984) 911.

[15] E.K. Sklyanin, J. Phys. A: Math. Gen. 21 (1988) 2375.

[16] L. Mezincescu, R.I. Nepomechi, J. Phys. A: Math. Gen. 24 (1991) L17;

L. Mezincescu, R.I. Nepomechi, Int. J. Mod. Phys. A 7 (1991) 5231;

L. Mezincescu, R.I. Nepomechi, Int. J. Mod. Phys. A 7 (1992) 5657. 
[17] H.Q. Zhou, X.Y. Ge, J.R. Links, M.D. Gould, Nucl. Phys. B 546 (1999) 779.

[18] G. Bedürftig, H. Frahm, Physica E 4 (1999) 246;

G. Bedürftig, H. Frahm, J. Phys. A: Math. Gen. 32 (1999) 4585;

G. Bedürftig, H. Frahm, J. Phys. A: Math. Gen. 30 (1997) 4139;

G. Bedürttig , B. Brendel, H. Frahm, R.M. Noack, Phys. Rev. B 58 (1998) 10225.

[19] H. Asakawa, M. Suzuki, Physica A 236 (1997) 376;

H. Asakawa, Physica A 256 (1998) 229.

[20] H. Asakawa, M. Suzuki, J. Phys. A: Math. Gen. 29 (1996) 225;

M. Shiroishi, M. Wadati, J. Phys. Soc. Jpn. 66 (1997) 1.

[21] S. Skorik, A. Kapustin, J. Phys. A: Math. Gen. 29 (1996) 1629;

S. Skorik, H. Saleur, J. Phys. A: Math. Gen. 28 (1995) 6605.

[22] A.A. Zvyagin, Phys. Rev. B 60 (1999) 15266;

A.A. Zvyagin, P. Schlottmann, Phys. Rev. B 56 (1997) 300;

A.A. Zvyagin, H. Johannesson, Phys. Rev. Lett. 81 (1998) 2751.

[23] A. Foerster, M. Karowski, Nucl. Phys. B 408 (1993) 512;

A.J. Bracken, X.Y. Ge, Y.Z. Zhang, H.Q. Zhou, Nucl. Phys. B 516 (1998) 588;

X.-W. Guan, J. Phys. A: Math. Gen. 33 (2000) 5391.

[24] H.Q. Zhou, Phys. Rev. B 54 (1996) 41;

X.-W. Guan, M.-S. Wang, S.-D. Yang, Nucl. Phys. B 485 (1997) 685;

M. Shiroishi, M. Wadati, J. Phys. Soc. Jpn. 66 (1997) 2288;

T. Deguchi, R. Yue, K. Kusakabe, J. Phys. A: Math. Gen. 31 (1998) 7315.

[25] Z.-N. Hu, F.-C. Pu, Nucl. Phys. B 546 (1999) 691;

H. Fan, M. Wadati, X.-M. Wang, Phys. Rev. B 61 (2000) 3450;

A. Lima-Santos, Nucl. Phys. B 558 (1999) 637.

[26] H.-Q. Zhou, Phys. Rev. B 53 (1996) 5098;

A. Foerster, X.-W. Guan, J. Links, I. Roditi, H.-Q. Zhou, Nucl. Phys. B 596 (2001) 525.

[27] H.-Q. Zhou, D.-M. Tong, Phys. Lett. A 232 (1997) 377.

[28] A.J. Bracken, X.-Y. Ge, Y.-Z. Zhang, H.-Q. Zhou, Nucl. Phys. B 516 (1998) 603.

[29] H.J. de Vega, A. González-Ruiz, J. Phys. A: Math. Gen. 26 (1993) L519;

Mod. Phys. Lett. A 9 (1994) 2207;

J. Abad, M. Rios, Phys. Lett. B 352 (1995) 92. 